\title{
Parallel Robot Translational Performance Evaluation through Direction-Selective Index (DSI)
}

\author{
G. Boschetti, R. Rosa, and A. Trevisani \\ Dipartimento di Tecnica e Gestione dei Sistemi Industriali, Università degli Studi di Padova, Stradella S. Nicola 3, \\ 36100 Vicenza, Italy \\ Correspondence should be addressed to A. Trevisani, alberto.trevisani@unipd.it
}

Received 28 January 2011; Revised 13 May 2011; Accepted 26 May 2011

Academic Editor: Yuan Zheng

Copyright ( 2011 G. Boschetti et al. This is an open access article distributed under the Creative Commons Attribution License, which permits unrestricted use, distribution, and reproduction in any medium, provided the original work is properly cited.

Performance indexes usually provide global evaluations of robot performances mixing their translational and/or rotational capabilities. This paper proposes a definition of performance index, called direction-selective index (DSI), which has been specifically developed for parallel manipulators and can provide uncoupled evaluations of robot translational capabilities along relevant directions. The DSI formulation is first presented within a general framework, highlighting its relationship with traditional manipulability definitions, and then applied to a family of parallel manipulators (4-RUU) of industrial interest. The investigation is both numerical and experimental and allows highlighting the two chief advantages of the proposed DSIs over more conventional manipulability indexes: not only are DSIs more accurate in predicting the workspace regions where manipulators can best perform translational movements along specific directions, but also they allow foreseeing satisfactorily the dynamic performance variations within the workspace, though being purely kinematic indexes. The experiments have been carried out on an instrumented 4-RUU commercial robot.

\section{Introduction}

Performance evaluation is one of the most important issues in the analysis and design of manipulators. Indeed, performance indexes may provide useful hints in the design and optimization of robots. In 1982, Salisbury and Craig firstly proposed the condition number of the transpose of the Jacobian matrix $\mathbf{J}$ as a measure of the workspace quality [1]. Some years later, Yoshikawa introduced the concept of manipulability, based on the Jacobian matrix, in order to find the best postures for manipulators [2]. Subsequently, in [3], Gosselin and Angeles suggested using the global conditioning index in order to evaluate the dexterity of a robot over a given workspace. Such an index is basically an integral mean of the condition number discussed in [1]. In [4], the definitions of manipulability, condition number, and dexterity index have been revisited in order to be applied to parallel robots. Novel formulations have been proposed, which are different from the ones introduced in [1-3].

Performance indexes based on the Jacobian matrix may be affected by the presence of inhomogeneities in the velocity ratios that may lead to ineffective results. Such a problem was found in [5] in the analysis of the Jacobian matrix of serial manipulators. It was solved by defining a characteristic length of the manipulator and by proposing a weighting positive matrix that allows obtaining a homogenous Jacobian. Unfortunately, such a solution cannot be easily extended to parallel manipulators. The first formulation of a dimensionally homogeneous Jacobian matrix overcoming this problem for planar parallel manipulators was introduced in [6]. Such a Jacobian relates the actuator velocities to the ones of the Cartesian coordinates of two points of the mobile platform. Following such a reasoning, in [7], a new dimensionally homogeneous Jacobian matrix was formulated by relating the velocities of three points (coplanar with the mobile platform joints) to the actuator ones. The same approach has been proposed in [8] for force transmission analysis, and then it has been reformulated in [9] for the dexterity analysis of parallel manipulators.

A simple performance index based on the Jacobian matrix has been also presented in [10]. The proposed index 
is derived by developing an upper bound on the norm of the rate of change of the Jacobian matrix. Such an upper bound has a significant role in a sufficiency condition for Jacobian rank preservation. It is proved that the index proposed in [9] provides information coherent with manipulability [2] and the condition number [1], while being easier to compute.

In [11], the dexterity index has been exploited together with a space utility ratio in order to compose a mixed performance index for optimizing translational parallel manipulators. The introduction of the space utility ratio index ensures avoiding disproportion between the reachable workspace volume and the structure of the robot by using the ratio between the total workspace volume and the physical size of the manipulator.

A performance investigation on translational parallel manipulators has been presented in [12]. The performance index is given by the minimum and the maximum eigenvalues of the stiffness matrix obtained from the Jacobian as proposed in [13]. The effectiveness of this index has been proved by applying it to an innovative parallel manipulator whose kinematics is analyzed in [14].

Successively, in $[15,16]$, a kinetostatic performance index, called power manipulability, has been introduced. Power manipulability is related to the power within the mechanism and is fully homogeneous, whether the manipulator contains active joints of different type, or the task space combines both translation and rotation motion.

A different approach for the computation of manipulator dexterity has been proposed in some recent studies $[17,18]$, where a generalized transmission index that can evaluate the motion/force transmissibility of fully parallel manipulators has been defined through virtual coefficients and not through Jacobian matrix. Contrary to the other indexes, such a transmission index is frame-free (i.e., its value does not depend on the reference frame in which it is computed). Moreover, it can be used to identify a good transmission workspace, where not only is the parallel manipulator effective in terms of motion/force transmission, but also far from its singularities.

All the aforementioned indexes provide global evaluations of robot performances mixing the translational capabilities of robots or the rotational ones. However, in [4], the concept of manipulability has been extended to evaluate the translational and rotational capabilities of parallel manipulators. In such a case, the Jacobian matrix has been split in two submatrices, $\mathbf{J}_{t}$ and $\mathbf{J}_{r}$, evaluated separately. Following a similar reasoning, in [19], it is proved that it is useful to split the Jacobian matrix $\mathbf{J}_{t}$ in order to extend the manipulability definition further and analyze the translational performance of a robot along a specific direction, which originated the concept of direction-selective performance index (DSI). In [19], DSI formulation has been adopted to study and compare the performances along different directions of a 3-UPU manipulator and a delta-like (4-RUU) manipulator. Generally speaking, when uncoupled DSIs are available for horizontal and vertical movements, they may be usefully adopted to foresee performances on those tasks (e.g., the pick-and-place task), which consist of continuous sequences of vertical and horizontal displacements. Additionally, DSIs may be usefully employed to optimize the location of target frames with respect to a robot, or of a robot within its workcell.

There are two main motivations for this paper:

(i) experimentally proving the practical usefulness of DSIs in predicting the regions within the workspace where a manipulator can best perform a specific movement;

(ii) showing that DSIs, though being purely kinematic indexes, allow foreseeing dynamic performance variations within the workspace satisfactorily.

In order to prove the practical usefulness of DSIs, in this work, their formulation is applied to foresee performance dissimilarities in the vertical and horizontal movements of an industrial parallel manipulator belonging to the family of four-leg delta-like (4-RUU) manipulators: the Adept Quattro. Additionally, a comparative study is performed between the results obtained by applying DSIs and manipulability. The DSI formulation adopted in this paper is more general than the one first introduced in [19], while several mathematical formulations of manipulability are considered. The analyses made are both numerical and experimental.

The experimental investigation has been done mainly because the numerical results highlight major differences in the best performance regions identified by the DSIs and the manipulability indexes. The experiments have been carried out by instrumenting the robot with an MEMS accelerometer and by making the robot perform extensive tests consisting in constant-length vertical and horizontal movements of the moving platform within the whole workspace: the expected and the real performances of the robot have then been compared. The results from the experimental investigation prove the validity of the hints inferred through the proposed DSI formulation and hence its practical usefulness. In particular, as theoretically expected, DSIs turn up to be far more descriptive than manipulability.

The rest of the paper is set out as follows. Section 2 provides the definition of DSI within a general framework. Then, in Section 3, the DSI definition is applied to the family of 4-RUU parallel manipulators. Section 4 shows the results of a numerical investigation aimed at assessing and comparing the shapes of the DSIs and of manipulability indexes within the workspace of the Quattro robot. Planar and spatial graphical representations of the shapes of the indexes are provided. The experimental campaign and its results are then presented and discussed in Section 5. Finally, Section 6 draws the conclusions.

\section{Definition of Direction-Selective Index}

The congruence equation of the position analysis of a parallel manipulator can be generally defined as $\mathbf{F}(\mathbf{q}, \mathbf{x})=\mathbf{0}$, where $\mathbf{q}$ denotes the joint variable vector, $\mathbf{x}$ is the generalized coordinates of the end effector, $\mathbf{F}$ is an $n$-dimensional implicit function of $\mathbf{q}$ and $\mathbf{x}$, and $\mathbf{0}$ is the $n$-dimensional zero vector. By differentiating the congruence equation with respect to 
time, the velocity relation $\mathbf{J}_{x} \cdot \dot{\mathbf{x}}-\mathbf{J}_{q} \cdot \dot{\mathbf{q}}=\mathbf{0}$ is achieved, where $\mathbf{J}_{x}=\partial f_{i} / \partial x_{j}$ and $\mathbf{J}_{q}=-\partial f_{i} / \partial q_{k}$.

The two matrices $\mathbf{J}_{x}$ and $\mathbf{J}_{q}$ are the $n \times n$ Jacobian matrices of, respectively, the end-effector coordinates and the joint variables. Such matrices obviously depend on the configuration of the manipulator. From these equations, there immediately follow the expressions of $\mathbf{J}:=\mathbf{J}_{x}^{-1} \cdot \mathbf{J}_{q}$ and $\mathbf{J}^{-1}:=\mathbf{J}_{q}^{-1} \cdot \mathbf{J}_{x}$ and also the solutions of the forward $(\dot{\mathbf{x}}=\mathbf{J} \cdot \dot{\mathbf{q}})$ and of the inverse $\left(\dot{\mathbf{q}}=\mathbf{J}^{-1} \cdot \dot{\mathbf{x}}\right)$ velocity kinematic problems.

In parallel robotics, forward position kinematics is usually harder to solve than inverse kinematics. Sometimes the analytical solution of the forward problem is not even available. Hence, the analytical expression of the inverse Jacobian matrix $\mathbf{J}^{-1}$ is usually easier to achieve than $\mathbf{J}$.

One of the most popular performance indexes is the manipulability index defined by Yoshikawa in [2] as follows:

$$
\mu=\sqrt{\operatorname{det}\left(\mathbf{J} \cdot \mathbf{J}^{\mathrm{T}}\right)} .
$$

Such an index was conceived for serial robots, hence it is based on the Jacobian matrix of the forward kinematic problem. However, as previously discussed, in parallel manipulators, the inverse Jacobian matrix is usually available, which suggests introducing some small changes to the index definition. By recalling that if $\lambda_{i}$ are the eigenvalues of a generic square matrix, the eigenvalues of its inverse are $1 / \lambda_{i}$, and that the determinant of a matrix is equal to the dot product of its eigenvalues, a definition of the manipulability more suitable to parallel manipulators can be inferred

$$
\mu=\frac{1}{\sqrt{\operatorname{det}\left(\mathbf{J}^{-\mathrm{T}} \cdot \mathbf{J}^{-1}\right)}} .
$$

By making use of such a definition, the values of $\mu$ become comparable with those computed through Yoshikawa's formulation and become identical to such values in case of square Jacobian matrices. Moreover, the values taken by $\mu$ are coherent with the singularity analysis for parallel robots defined in [20].

Other measure criteria of manipulability can be used in the performance analysis of parallel manipulators [4]; manipulability can indeed be also defined by means of

(i) the Euclidean norm (or Frobenius norm) $\left\|\mathbf{J} \cdot \mathbf{J}^{\mathrm{T}}\right\|_{F}=$ $\sqrt{\sum_{i, j=1}^{n}\left|a_{i j}\right|^{2}}$

(ii) the infinity norm $\left\|\mathbf{J} \cdot \mathbf{J}^{\mathrm{T}}\right\|_{\infty}=\max _{1 \leq i \leq m} \sum_{j=1}^{n}\left|a_{i j}\right|$,

where $a_{i j}$ are the elements of the matrix $\mathbf{J} \cdot \mathbf{J}^{\mathrm{T}}$. By following a reasoning similar to the one leading to (2) from (1), the following further definitions of manipulability can be provided for parallel manipulators:

$$
\begin{aligned}
\mu^{F} & =\frac{1}{\left\|\mathbf{J}^{-\mathrm{T}} \cdot \mathbf{J}^{-1}\right\|_{F}}, \\
\mu^{\infty} & =\frac{1}{\left\|\mathbf{J}^{-\mathrm{T}} \cdot \mathbf{J}^{-1}\right\|_{\infty}} .
\end{aligned}
$$

In order to try discriminating the performances related to linear and rotational displacements of the end effector of a robot, the Jacobian matrix may be split into two parts, $\mathbf{J}_{t}$ and $\mathbf{J}_{r}\left(\mathbf{J}=\left[\mathbf{J}_{t} \mid \mathbf{J}_{r}\right]^{\mathrm{T}}\right)$, which can be evaluated separately [4]. The submatrix $\mathbf{J}_{t}$ contains the ratios between the end-effector translations and the joint displacements; the submatrix $\mathbf{J}_{r}$ contains the ratios between the end-effector rotations and the joint displacements. Hence, different performance indexes can be computed making use of $\mathbf{J}_{t}$ and $\mathbf{J}_{r}$. Of course, such indexes still provide a global evaluation of the performances mixing either the translational capabilities of robot in the three directions or the rotational ones.

When parallel robots are considered, $\mathbf{J}_{t}$ and $\mathbf{J}_{r}$ are more easily computed through inverse kinematics; consider

(i) the matrix $\mathbf{J}_{x t}$, submatrix of $\mathbf{J}_{x}$ comprising the partial derivates with respect to the translational coordinates of the end effector,

(ii) the matrix $\mathbf{J}_{x r}$, submatrix of $\mathbf{J}_{x}$ comprising the partial derivates with respect to the rotational coordinates of the end effector.

In the following (4), the matrices $\mathbf{J}_{t}^{-1}$ and $\mathbf{J}_{r}^{-1}$ are then the inverse matrices for the translational and the rotational inverse velocity analyses:

$$
\mathbf{J}_{t}^{-1}=\mathbf{J}_{q}^{-1} \cdot \mathbf{J}_{x t}, \quad \mathbf{J}_{r}^{-1}=\mathbf{J}_{q}^{-1} \cdot \mathbf{J}_{x r} .
$$

The analyses based on the Jacobian submatrix $\mathbf{J}_{t}^{-1}$ and the values of the performance indexes that may be computed from such a matrix are usually affected by the units adopted to measure the end-effector velocities and the joint ones. Some "normalization" criteria hence need to be adopted to make the results of the analyses comparable regardless of the features of the manipulator active joints (see $[7,20]$ ). As a matter of fact, the coefficients of $\mathbf{J}_{t}^{-1}$ are the ratios between the linear velocities of the end effector and the velocities of the active joints, which might be either translational or rotational. In case of fully translational joints, the analysis of the matrix $\mathbf{J}_{t}^{-1}$ can be directly performed and leads to dimensionless indexes. Alternatively, if active joints are rotational, in order to keep the performance indexes dimensionless, it may be appropriate to modify the elements of matrix $\mathbf{J}_{t}^{-1}$ so as to relate the end-effector translational velocities to the tangent velocities of the drive cranks, rather than to their angular velocities. A new dimensionless matrix $\mathbf{J}_{D t}^{-1} \mathrm{can}$ therefore be adopted when the active joints are rotational; $\mathbf{J}_{D t}^{-1}$ is obtained by premultiplying $\mathbf{J}_{t}^{-1}$ by a diagonal matrix $\mathbf{D}$ made up of the lengths of the drive cranks.

$$
\mathbf{J}_{D t}^{-1}=\mathbf{D} \cdot \mathbf{J}_{t}^{-1}, \quad \mathbf{J}_{D t}=\mathbf{J}_{t} \cdot \mathbf{D}^{-1} .
$$

By replacing $\mathbf{J}^{-1}$ (and its transpose matrix) in (2) and (3), with $\mathbf{J}_{t}^{-1}$ or $\mathbf{J}_{D t}^{-1}$ (and its transpose matrix), according to the features of the active joints, it is possible to get the manipulability indexes referring to end-effector translational degrees of freedom. In particular, in case of fully rotational 
active joints, it holds

$$
\begin{gathered}
\mu_{t}=\frac{1}{\sqrt{\operatorname{det}\left(\mathbf{J}_{D t}^{-\mathrm{T}} \cdot \mathbf{J}_{D t}^{-1}\right)}}, \\
\mu_{t}^{F}=\frac{1}{\left\|\mathbf{J}_{D t}^{-\mathrm{T}} \cdot \mathbf{J}_{D t}^{-1}\right\|_{F}}, \\
\mu_{t}^{\infty}=\frac{1}{\left\|\mathbf{J}_{D t}^{-\mathrm{T}} \cdot \mathbf{J}_{D t}^{-1}\right\|_{\infty}} .
\end{gathered}
$$

The idea behind direction-selective indexes (DSIs) relies on splitting matrices $\mathbf{J}_{t}^{-1}$ or $\mathbf{J}_{D t}^{-1}$ into three column vectors in order to extend the manipulability formulation further and make it suitable to analyze robot performances along specific directions. As an example, the following holds in case of fully rotational active joints:

$$
\mathbf{J}_{D t}^{-1}=\left[\mathbf{J}_{X}^{-1}\left|\mathbf{J}_{Y}^{-1}\right| \mathbf{J}_{Z}^{-1}\right] .
$$

DSIs can then be straightforwardly obtained by replacing $\mathbf{J}^{-1}$ (and its transpose matrix) in (2) with $\mathbf{J}_{X}^{-1}, \mathbf{J}_{Y}^{-1}$, or $\mathbf{J}_{Z}^{-1}$ (and their transpose vectors)

$$
\begin{aligned}
& \mu_{X}=\frac{1}{\sqrt{\operatorname{det}\left(\mathbf{J}_{X}^{-\mathrm{T}} \cdot \mathbf{J}_{X}^{-1}\right)}}=\left(\mathbf{J}_{X}^{-\mathrm{T}} \cdot \mathbf{J}_{X}^{-1}\right)^{-1 / 2}, \\
& \mu_{Y}=\frac{1}{\sqrt{\operatorname{det}\left(\mathbf{J}_{Y}^{-\mathrm{T}} \cdot \mathbf{J}_{Y}^{-1}\right)}}=\left(\mathbf{J}_{Y}^{-\mathrm{T}} \cdot \mathbf{J}_{Y}^{-1}\right)^{-1 / 2}, \\
& \mu_{Z}=\frac{1}{\sqrt{\operatorname{det}\left(\mathbf{J}_{Z}^{-\mathrm{T}} \cdot \mathbf{J}_{Z}^{-1}\right)}}=\left(\mathbf{J}_{Z}^{-\mathrm{T}} \cdot \mathbf{J}_{Z}^{-1}\right)^{-1 / 2} .
\end{aligned}
$$

The DSIs $\mu_{X}, \mu_{Y}$, and $\mu_{Z}$ are here proposed to evaluate independently the translational capabilities of a manipulator along the axes of its world reference frame. The performances along any other relevant direction can be straightforwardly evaluated by suitably rotating the world reference frame, so that one axis coincides with the selected direction.

\section{DSI Formulation for 4-RUU Parallel Manipulators}

In order to assess the capability of the proposed indexes to foresee manipulator performance variations along selected directions, the definitions just provided for $\mu_{X}, \mu_{Y}$, and $\mu_{Z}$ have been applied to a generic 4-RUU parallel manipulator.

4-RUU manipulators are four-leg and four-degree-offreedom parallel manipulators conceived for performing high-speed and high-acceleration pick-and-place operations. The kinematic and dynamic models for this family of parallel robots are reported in [21]. Due to their architecture, these robots can produce Schöenflies motions (i.e., translations along the $X, Y$ and $Z$ direction and a rotation about the $Z$ axis), which are required in most pick-and-place operations, including packaging, picking, packing, and palletizing tasks. An example of 4-RUU manipulator is sketched in Figure 1, which also shows some essential geometrical parameters of the robot, of its workspace, and the robot world reference frame. The robot moving platform takes the shape of a rhombus whose side has length $2 d$. The platform is connected to the fixed base by four identical RUU serial chain legs, that is, legs with one revolute (R) and two universal (U) joints (see Figure 1).

Let $\left\{x_{0}, y_{0}, z_{0}, \theta_{0}\right\}^{\mathrm{T}}$ be the end-effector pose, and $\left\{\varphi_{1}, \varphi_{2}, \varphi_{3}, \varphi_{4}\right\}^{\mathrm{T}}$ the active joint coordinates. In order to compute the performance indexes for the studied family of parallel manipulators, since the active joints are all rotational, the following formulation of the translational matrix $\mathbf{J}_{D t}$ is used:

$$
\mathbf{J}_{D t}^{-1}=\mathbf{J}_{q}^{-1} \cdot \mathbf{D} \cdot \mathbf{J}_{x t} .
$$

Matrix $\mathbf{J}_{q}^{-1}$ is the inverse of the Jacobian matrix $\mathbf{J}_{q}$ and is defined as follows:

$$
\mathbf{J}_{q}^{-1}=\left[\begin{array}{cccc}
\frac{1}{j_{q_{1}}} & 0 & 0 & 0 \\
0 & \frac{1}{j_{q_{2}}} & 0 & 0 \\
0 & 0 & \frac{1}{j_{q_{3}}} & 0 \\
0 & 0 & 0 & \frac{1}{j_{q_{4}}}
\end{array}\right],
$$

with:

$$
\begin{aligned}
j_{q_{i}}=b\left[z_{0} \cos \varphi_{i}+\left(a-x_{0}-\frac{d \sqrt{2}}{2}-d \cos \left(\frac{\pi}{4}-\theta_{0}\right)\right)\right. \\
\left.\times \sin \varphi_{i}\right] \quad(i=1,3), \\
j_{q_{j}=b}\left[z_{0} \cos \varphi_{j}+\left(a-y_{0}-\frac{d \sqrt{2}}{2}+d \sin \left(\frac{\pi}{4}-\theta_{0}\right)\right)\right. \\
\left.\quad \times \sin \varphi_{j}\right] \quad(j=2,4) .
\end{aligned}
$$

Matrix $\mathbf{J}_{x t}$ is the translational part of the Jacobian matrix $\mathbf{J}_{x}$ and is defined as follows:

$$
\mathbf{J}_{x t}=\left[\begin{array}{ccc}
x_{0}+A+B-C_{1} & y_{0}+D-B & z_{0}-E_{1} \\
x_{0}+A-B & y_{0}+D+B-C_{2} & z_{0}-E_{2} \\
x_{0}-A-B+C_{3} & y_{0}-D+B & z_{0}-E_{3} \\
x_{0}-A+B & y_{0}-D-B+C_{4} & z_{0}-E_{4}
\end{array}\right],
$$

where $A=d \cos \left((\pi / 4)-\theta_{0}\right), B=d(\sqrt{2} / 2), C_{k}=a+b \cos \varphi_{k}$, $D=d \sin \left((\pi / 4)-\theta_{0}\right)$, and $E_{k}=b \sin \varphi_{k}(k=1, \ldots, 4)$.

Finally, matrix $\mathbf{D}$ takes the following form: $\mathbf{D}=b \cdot \mathbf{I}_{4}$, where $\mathbf{I}_{4}$ is the identity matrix of size four and $b$ is the crank length. 


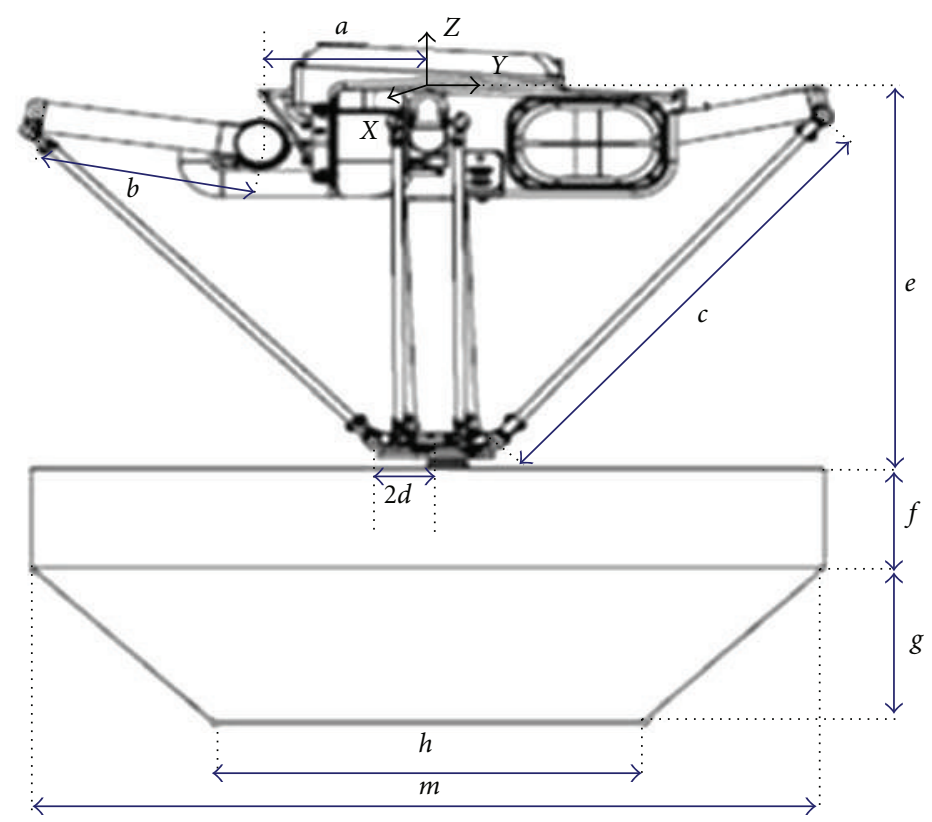

Figure 1: Schematic representation of a 4-RUU parallel robot and of its workspace.

The DSIs $\mu_{X}, \mu_{Y}$, and $\mu_{Z}$ can be computed through (8) by simply extracting the column vectors $\mathbf{J}_{X}^{-1}, \mathbf{J}_{Y}^{-1}$, and $\mathbf{J}_{Z}^{-1}$ from $\mathbf{J}_{D t}^{-1}$,

$$
\begin{gathered}
\mathbf{J}_{X}^{-1}=\mathbf{J}_{q}^{-1} \cdot\left\{x_{0}+A+B-C_{1}, x_{0}+A-B,\right. \\
\left.x_{0}-A-B+C_{3}, x_{0}-A+B\right\}^{\mathrm{T}} \cdot b, \\
\mathbf{J}_{Y}^{-1}=\mathbf{J}_{q}^{-1} \cdot\left\{y_{0}+D-B, y_{0}+D+B-C_{2}, y_{0}-D+B,\right. \\
\left.y_{0}-D-B+C_{4}\right\}^{\mathrm{T}} \cdot b, \\
\mathbf{J}_{Z}^{-1}=\mathbf{J}_{q}^{-1} \cdot\left\{z_{0}-E_{1}, z_{0}-E_{2}, z_{0}-E_{3},\right. \\
\left.z_{0}-E_{4}\right\}^{\mathrm{T}} \cdot b .
\end{gathered}
$$

\section{Numerical Investigation on an Industrial 4- RUU Manipulator: DSI versus Manipulability}

A numerical investigation has been carried out by applying the theory developed above to an industrial 4-RUU manipulator (the Adept Quattro). The goal of the investigation is to point out the different suggestions provided the manipulability indexes, in terms of regions of the workspace where the robot performances should be optimal. In order to achieve exhaustive predictions of the robot performances over its full workspace (shown in Figure 1), it has been necessary to discretize the workspace through a regular and thick grid. The grid has been constructed by selecting 12 equally spaced horizontal planes, located at $z_{u}(u=1, \ldots, 12)$ in the robot world reference frame. Half the planes belong to the upper cylindrical region of the workspace, and half to the lower truncated conic region. Each plane contains 11 concentric circles of radius $r_{v}(v=1, \ldots, 11)$. Each circle is split into 36 points with polar angle $\theta_{w}(w=1, \ldots, 36)$. Hence, the grind comprises 396 points spread through the entire volume of the workspace.

The cylindrical coordinates of each point $P$ of the grid have been transformed into Cartesian coordinates in the robot world frame using the following basic expressions:

$$
\begin{gathered}
x_{P}=r_{v} \cos \theta_{w}, \\
y_{P}=r_{v} \sin \theta_{w}, \\
z_{P}=z_{u} .
\end{gathered}
$$

The performance indexes in all the grid points have then been computed by setting $x_{0}=x_{P}, y_{0}=y_{P}$, and $z_{0}=z_{P}$. The rotation of the end effector $\left(\theta_{0}\right)$ has instead been set equal to zero in all the grid points.

Table 1 collects the chief geometrical parameters of the Quattro robot and of its workspace (refer to Figure 1 for the meaning of each parameter).

Figures 2 through 7 show the values taken by the manipulability indexes $\mu_{t}$ (Figure 2), $\mu_{t}^{F}$ (Figure 3), and $\mu_{t}^{\infty}$ (Figure 4) and by the DSIs $\mu_{X}$ (Figure 5), $\mu_{Y}$ (Figure 6), and $\mu_{Z}$ (Figure 7 ) over the robot workspace.

In these figures, a spatial view of the shapes of the indexes is given. So as to simplify the comparison among the predictions of the indexes, regardless of each index values and range of variation, the best performance regions take dark red color in all the figures, while the worst performance regions take dark blue color.

It may be immediately recognized that the manipulability indexes $\mu_{t}, \mu_{t}^{F}$, and $\mu_{t}^{\infty}$ have similar shapes; these indexes depict spherical-like and concentric isomanipulability surfaces. The indexes always take their best values in the inner regions of the workspace, which are hence identified as 


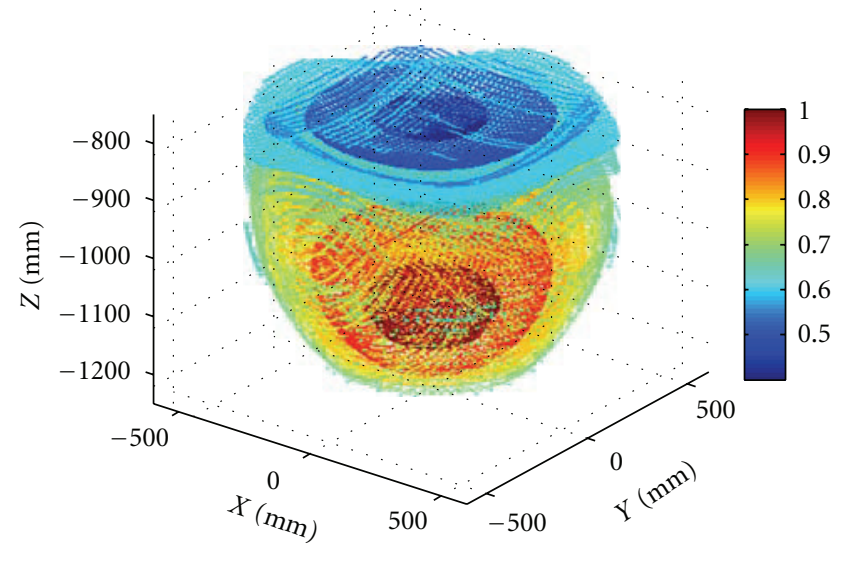

Figure 2: Manipulability index $\mu_{t}$.

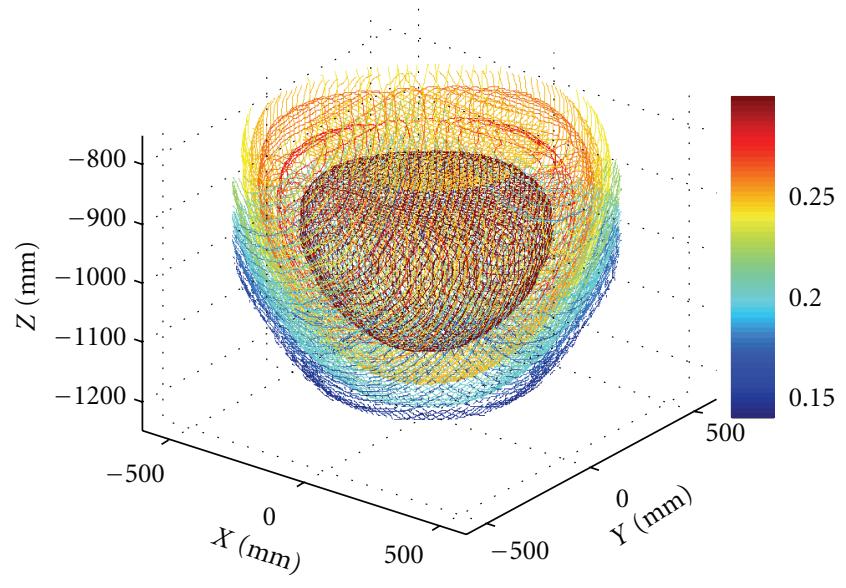

FIGURE 3: Manipulability index $\mu_{t}^{F}$.

TABLE 1: Quattro robot geometric and workspace parameters.

\begin{tabular}{lc}
\hline Symbols & Values $(\mathrm{mm})$ \\
\hline$a$ & 265 \\
$b$ & 370 \\
$c$ & 830 \\
$d$ & 80 \\
$e$ & 780 \\
$f$ & 220 \\
$g$ & 180 \\
$h$ & 700 \\
$m$ & 1140 \\
\hline
\end{tabular}

the best performance regions. The vertical position of such regions depends on the formulation chosen. The index values then decrease in the regions closer to the boundaries of the workspace. The shape shown by the manipulability indexes is rather intuitive; the best manipulator performances seems to be achievable close to the center of the manipulator workspace. However, considerably different hints come from the DSIs $\mu_{X}, \mu_{Y}$, and $\mu_{Z}$, that is, when considering

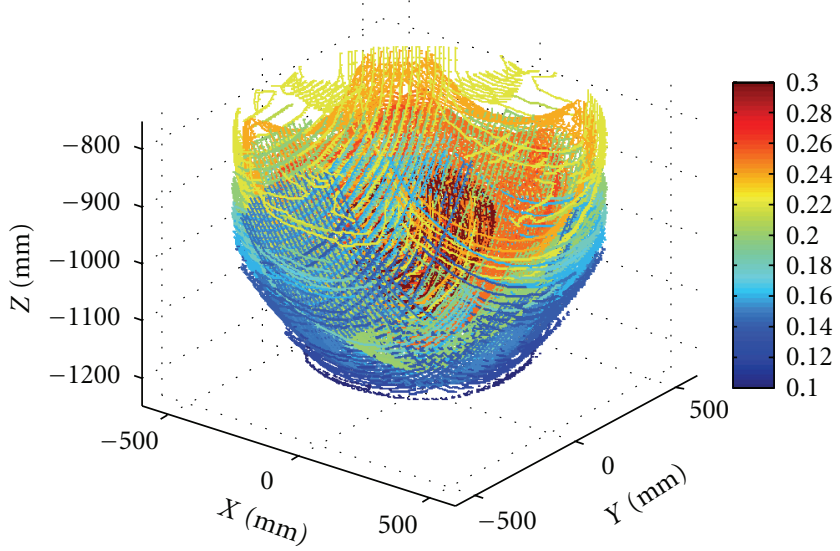

FIgURE 4: Manipulability index $\mu_{t}^{\infty}$.

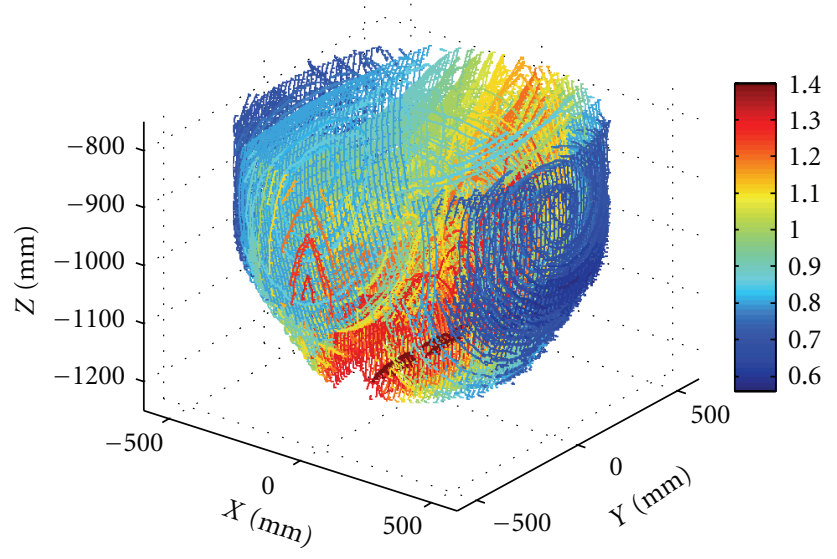

FIgURE 5: DSI $\mu_{X}$.

the expected performances for translations along different directions.

Let us first consider $\mu_{X}$ and $\mu_{Y}$ (Figures 5 and 6), whose shapes are nearly identical, apart from a rotation by $\pi / 2$ radians about the $Z$ axis. The isomanipulability regions are no longer spherical like and concentric, but rather half-oval in the best performance regions and elliptical like elsewhere. Additionally, the best performance regions are not located at the center of the workspace, but they are close to the boundaries and symmetrical across the axis orthogonal to the selected direction (e.g., orthogonal to axis $Y$ in the case of $\mu_{X}$; see Figure 5 ). The worst performance regions are instead the ones which are furthest from the symmetry axis.

As far as $\mu_{Z}$ is concerned, Figure 7 shows that the best values of the index are found in a ring like region surrounding the upper part of the workspace. Less satisfactory values are instead computed for the lower part of the workspace.

A better understanding of the shapes of these indexes can be obtained by plotting the values they take on some significant sample planes within the workspace. A few examples are shown in the Figures from 8 through 12, where isomanipulability curves are shown. These figures allow highlighting better the differences between DSIs and 


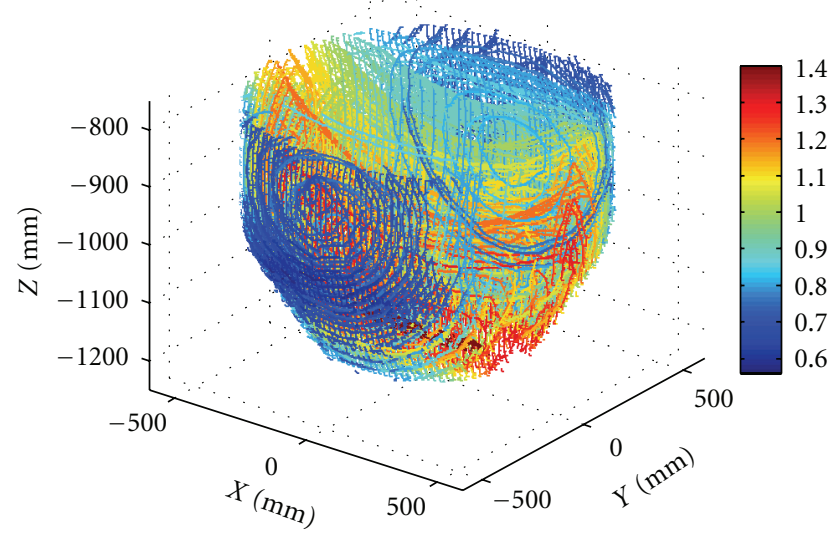

FIGURE 6: DSI $\mu_{Y}$.

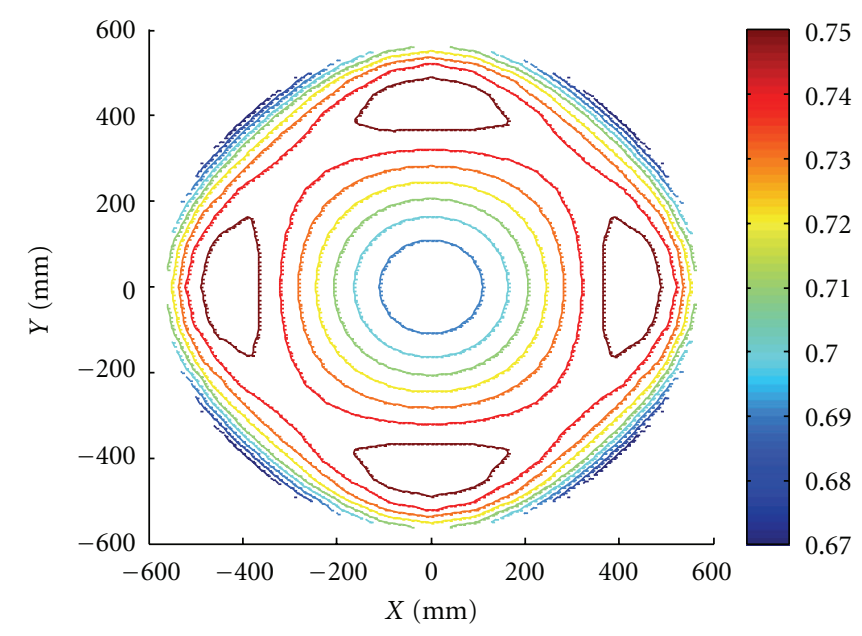

Figure 8: Manipulability index $\mu_{t}$ in the $X Y$ plane $(Z=-900)$.

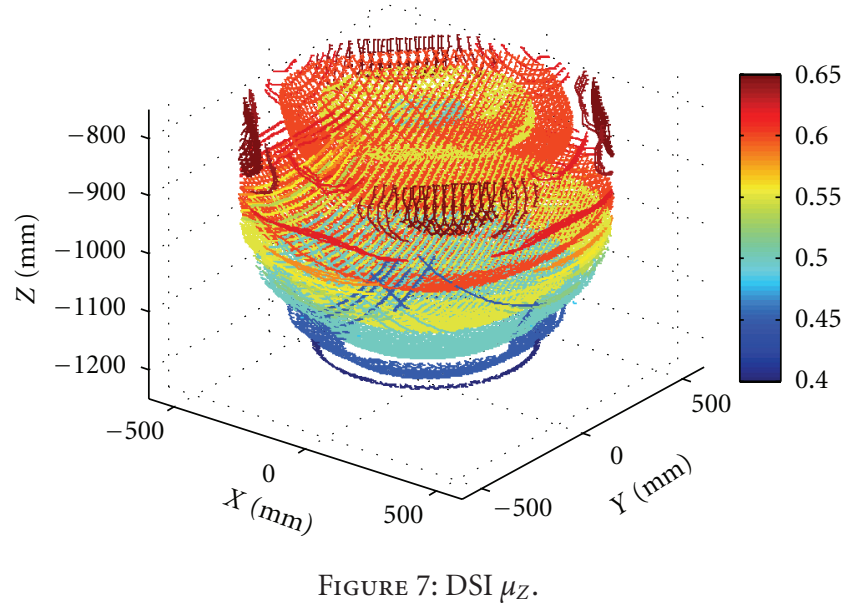

manipulability. Since the shapes of the three manipulability indexes are alike (see Figures 2, 3, and 4) and are very different from the ones of the DSIs, in the following analyses and figures only the manipulability index $\mu_{t}$ will be compared with the DSIs.

Figures 8,9 , and 10 show the values taken in the $X Y$ plane with coordinate $Z=-900$ by the manipulability index $\mu_{t}$, and by the DSIs $\mu_{X}$ and $\mu_{Y}$. Such figures clarify the abovementioned differences between the best performance region predictions. It is apparent that the use of $\mu_{t}$ seems to lead to conclusions that are misleading: for example, the existence of four best performance regions rotated $\pi / 2$ degrees with respect to each other and is in contrast with the results provided by the DSIs, where two best performance regions rotated $\pi$ degrees with respect to each other, appears close to the workspace boundaries and symmetrically displaced across the direction orthogonal to the selected direction of motion.

The values taken in the $Y Z$ plane with coordinate $X=0$ by $\mu_{t}$ and by the DSI $\mu_{Z}$ are instead plotted in Figures 11 and 12. The manipulability index $\mu_{t}$ (Figure 11) reaches its best

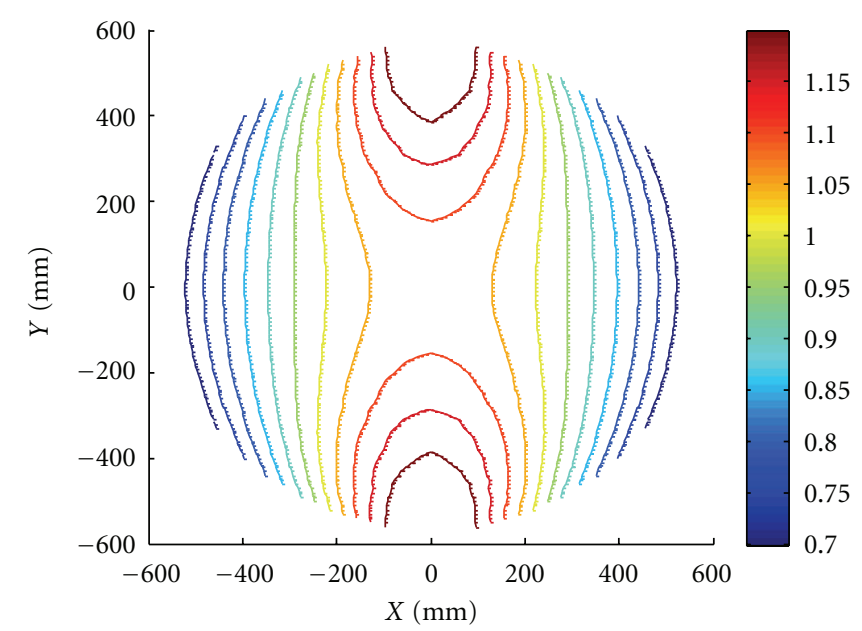

FIgURE 9: DSI $\mu_{X}$ in the $X Y$ plane $(Z=-900)$.

values in a region located close to the middle of the axis $Y$ and close to the bottom (i.e., to low values of $Z$ ) of the this planar slice of the workspace. The manipulability values decrease in the areas close to the boundaries of the workspace and take the less satisfactory values at the top of the workspace.

Conversely, Figure 12 shows that $\mu_{Z}$ takes its best values in a curved region crossing the planar slice of the workspace and achieving the very best values at the upper right and left boundaries. The region close to the bottom of workspace is where the less satisfactory values are computed.

\section{Experimental Validation}

The numerical results shown demonstrate that there are significant discrepancies between the predictions made by manipulability indexes and the DSIs. In order to assess experimentally which indexes provide the best predictions, some relevant experimental tests have been carried out on the Adept Quattro manipulator. 


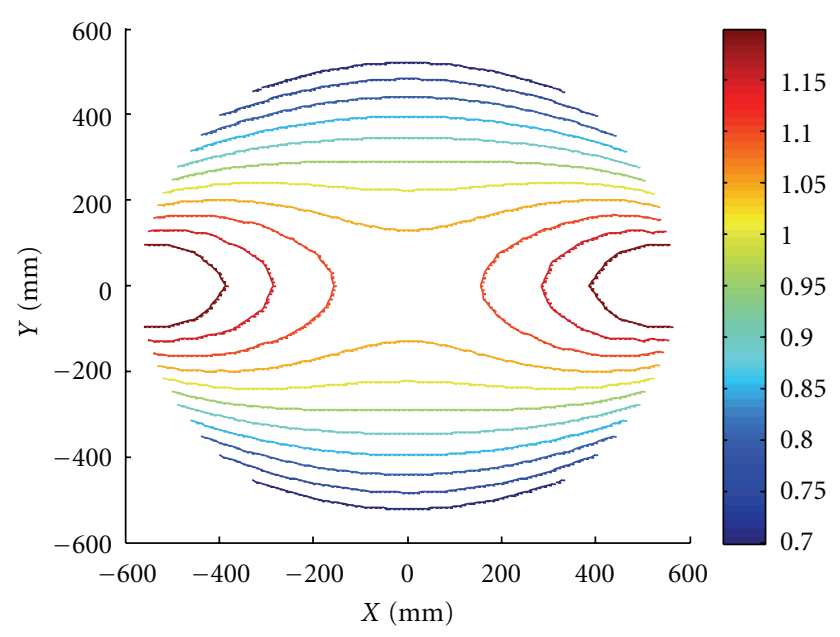

Figure 10: DSI $\mu_{Y}$ in the $X Y$ plane $(Z=-900)$.

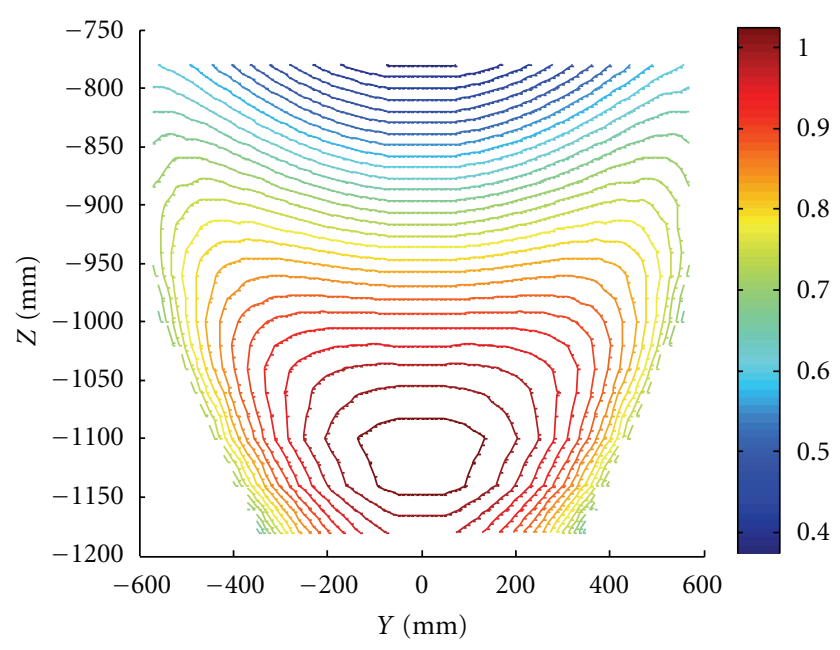

FIgURE 11: Manipulability index $\mu_{t}$ in the $Y Z$ plane $(X=0)$.

The experiments have been carried out with two objectives:

(i) evaluating the translational velocity performances of the robot moving platform within the workspace,

(ii) comparing the measured performance variations with the predictions inferred by the indexes analyzed.

Such a comparison makes sense given the apparent dependence on the Jacobian matrix elements of both the indexes and the translational velocities.

The moving platform of the Quattro robot has been instrumented with an uniaxial PCB MEMS accelerometer model 3741 (see Figure 13) alternatively employed to measure accelerations along the $X$ and the $Z$ axes of the robot world reference frame (see Figure 1). The adopted accelerometer has a measuring range of $\pm 30 \mathrm{G}$ and a frequency range between 0 (DC) and $2 \mathrm{kHz}$. An HBM MGC signal conditioner with ML10 modules has been used to power the MEMS accelerometer and to filter its output signals (a low-pass filter having a Butterworth characteristic

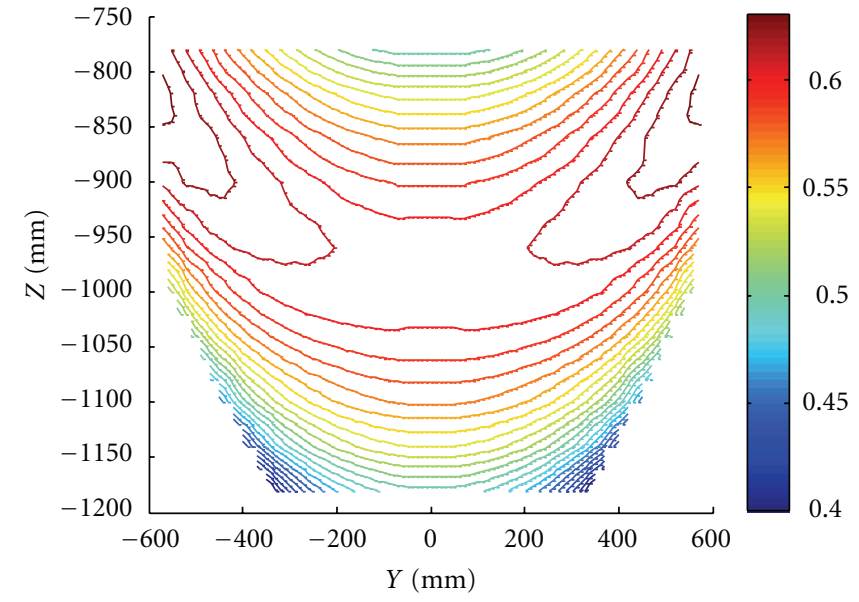

Figure 12: DSI $\mu_{Z}$ in the $Y Z$ plane $(X=0)$.

and a cut-off frequency of $200 \mathrm{~Hz}$ has been employed). The filtered data have been acquired and logged by means of an LMS Pimento analyzer. Finally, experimental data have been processed (high pass filtered and integrated numerically) and compared to the performance index values using MatlabSimulink.

The experimental tests have been carried out performing several repetitions of either straight horizontal displacements or straight vertical displacements in all the grid points into which the workspace has been split (see the grid definition in Section 4), and where performance indexes have already been computed.

As far as the Adept V+ software program written to run the robot is concerned, it is worth highlighting that

(i) the "continuous path" feature of the robot controller has been disabled to avoid modifications of the desired path during the manipulator motion;

(ii) the "speed" command has been set equal to $100 \%$ (i.e., no speed limitation has been introduced in path planning);

(iii) the sharpest acceleration profile (square-wave) has been adopted.

As far as the horizontal (along the $X$ direction) displacements are concerned, around each grid point, the endeffector has been made to perform a sequence of five $30 \mathrm{~mm}$ straight forward displacements and five $30 \mathrm{~mm}$ straight backwards displacements. The peak-to-peak amplitude of the displacements is $60 \mathrm{~mm}$. The midpoint of these displacements coincides with the grid point considered. The amplitude chosen for the displacements has been selected after some trials to attain two concurrent results: to let the robot achieve relevant velocities at the midpoints and to allow assessing the robot performances in an adequate number of grid points throughout the workspace. The repetition of identical displacements has been motivated by the need of checking the repeatability of the measured accelerations.

Similar tests have been carried out with vertical displacements (along the $Z$ direction): at each grid point, the end 


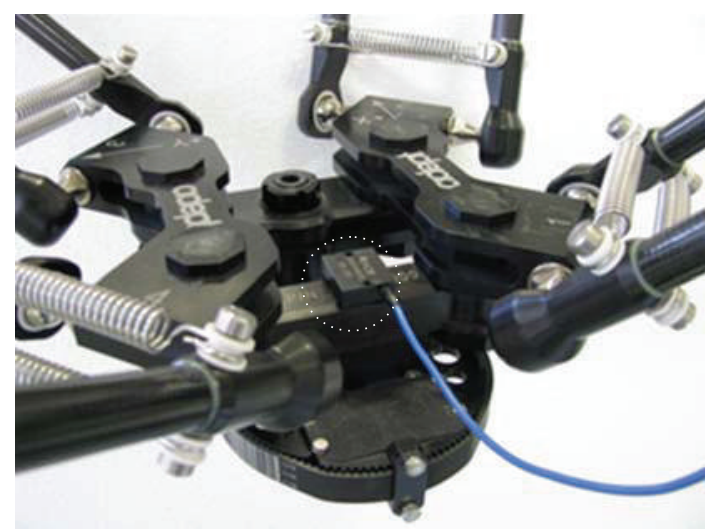

(a)

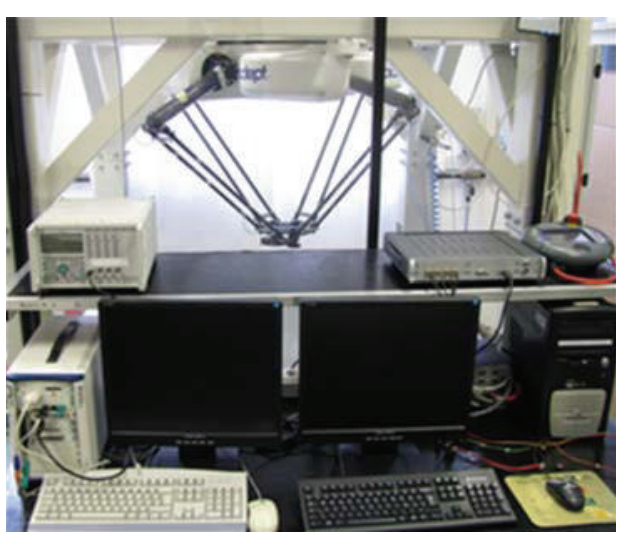

(b)

FIgURE 13: (a) The MEMS accelerometer (in the dotted circle) fixed on the moving platform. (b) The experimental setup.
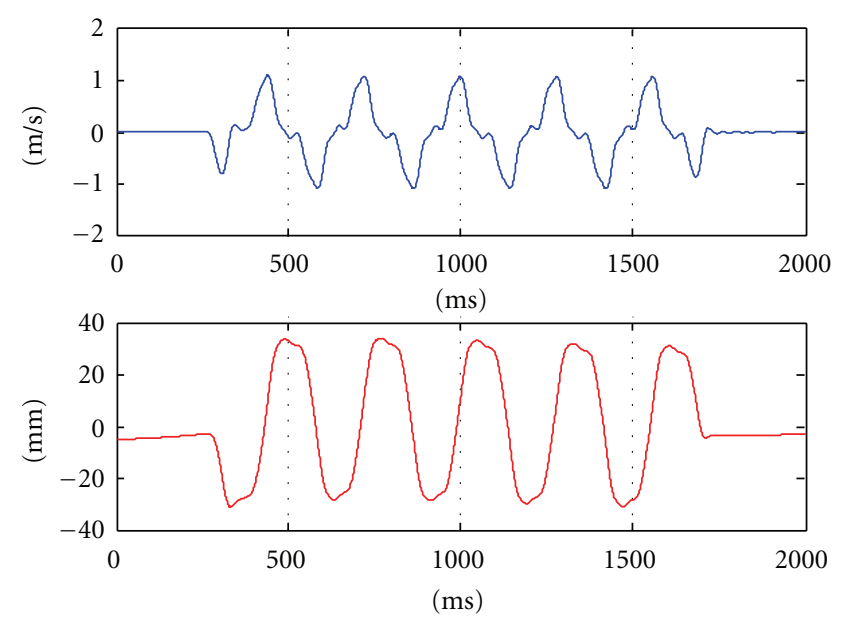

Figure 14: Sample horizontal velocities and displacements of the robot end effector measured at a grid point of the workspace.

effector has been made perform a sequence of six $30 \mathrm{~mm}$ straight upwards displacements and five $30 \mathrm{~mm}$ straight downward displacements. The peak-to-peak amplitude of the displacements is again $60 \mathrm{~mm}$, and the midpoint of these displacements coincides with the grid point considered. The acceleration data collected during the tests have been processed in order to gather the maximum horizontal and vertical velocity achieved by the end effector at each grid point. Maximum velocities are necessarily reached at the midpoints of the displacements (i.e., at the grid points of interests). As previously mentioned, velocities have been computed from accelerations by numerical integration. Signal detrending has been obtained by filtering the acceleration signals through a second-order Butterworth high-pass filter with passband frequency at $0.2 \mathrm{~Hz}$.

A sample plot of the horizontal velocities computed is shown in Figure 14. The same figure also shows the displacements computed through double integration; the mentioned $60 \mathrm{~mm}$ peak-to-peak amplitude of the displacements can be recognized. By comparing the two plots, it can be also
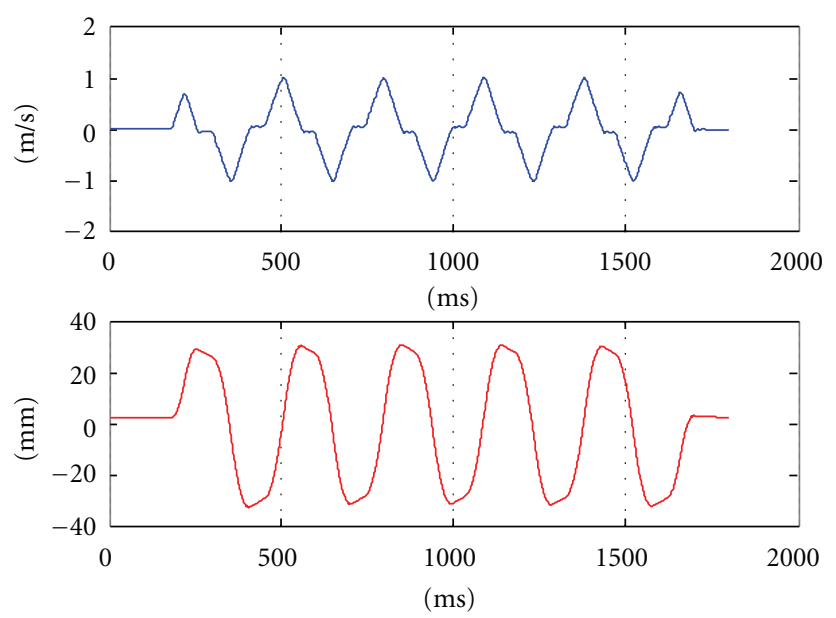

Figure 15: Sample vertical velocities and displacements of the robot end effector measured at a grid point of the workspace.

verified that maximum velocities are reached at about the midpoints of the paths. The same considerations hold for the vertical velocities (see Figure 15).

For each direction of motion (horizontal or vertical) and for each sequence of displacements (i.e., for each grid point), the very maximum absolute value of velocity achieved during the repetition of the displacements has then been detected and related to the grid point. For example, the subplots (b) of the Figures from 16 through 21 show the velocity values computed for the points belonging to three horizontal halfplanes $X Y$ and three vertical half-planes $Y Z$. The horizontal half-planes are located at $Z=-800 \mathrm{~mm}$ (Figure 16), $Z=$ $-900 \mathrm{~mm}$ (Figure 17), and $Z=-1050 \mathrm{~mm}$ (Figure 18). The vertical half-planes are instead located at $X=0 \mathrm{~mm}$ (Figure 19), $X=100 \mathrm{~mm}$ (Figure 20), and $X=200 \mathrm{~mm}$ (Figure 21). Velocity values have been normalized in order to take 1 as the maximum value.

The Figures from 16 through 21 also show the values taken by the studied performance indexes in the same planes; manipulability index $\mu_{t}$ is shown in the subplots (a) of the 


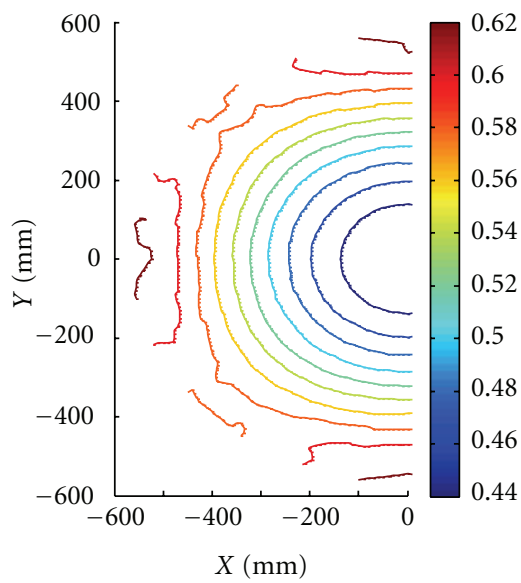

(a)

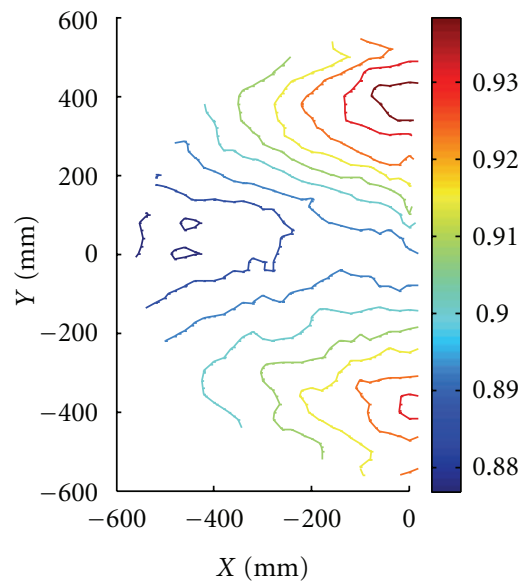

(b)

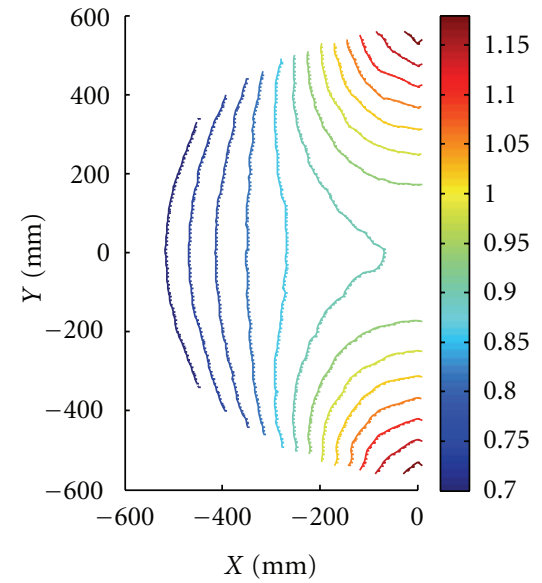

(c)

Figure 16: Manipulability index $\mu_{t}$ (a), normalized velocity along the $X$ direction (b), and DSI $\mu_{X}$ computed in the horizontal half-plane $X Y$ with $Z=-800 \mathrm{~mm}$ (c).

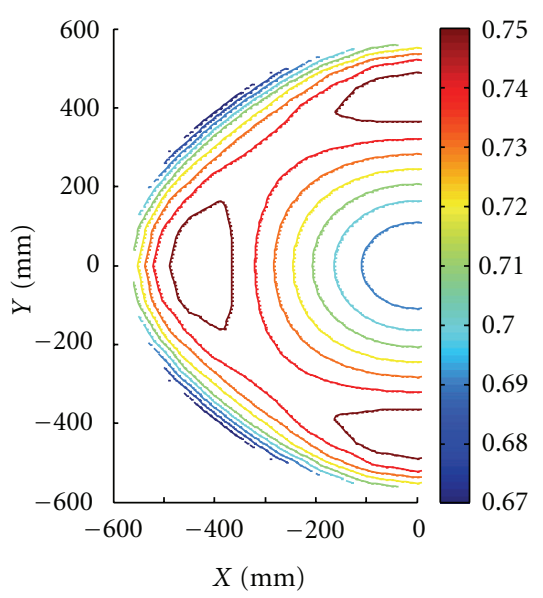

(a)

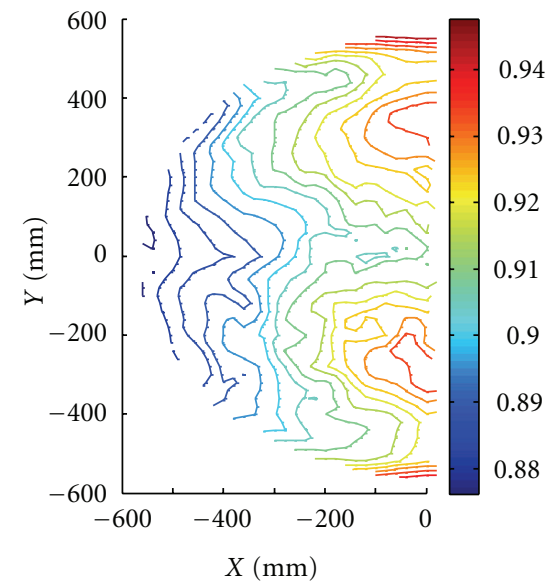

(b)

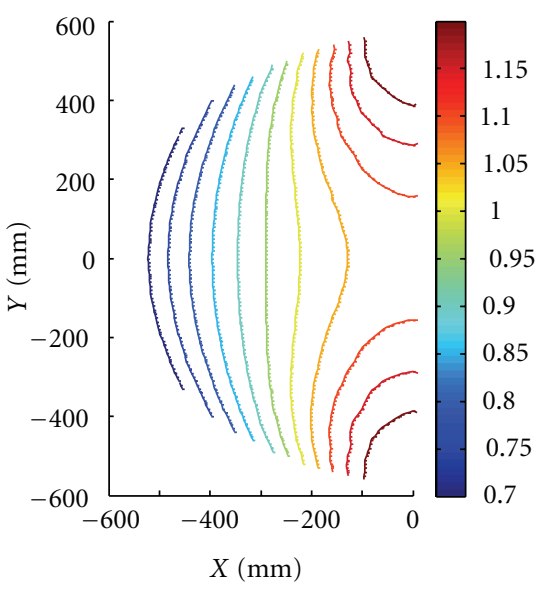

(c)

FIgURE 17: Manipulability index $\mu_{t}$ (a), normalized velocity along the $X$ direction (b), and DSI $\mu_{X}$ computed in the horizontal half-plane $X Y$ with $Z=-900 \mathrm{~mm}$ (c).

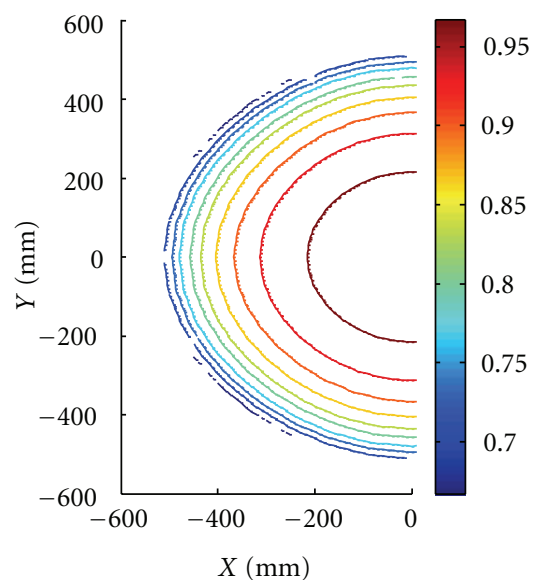

(a)

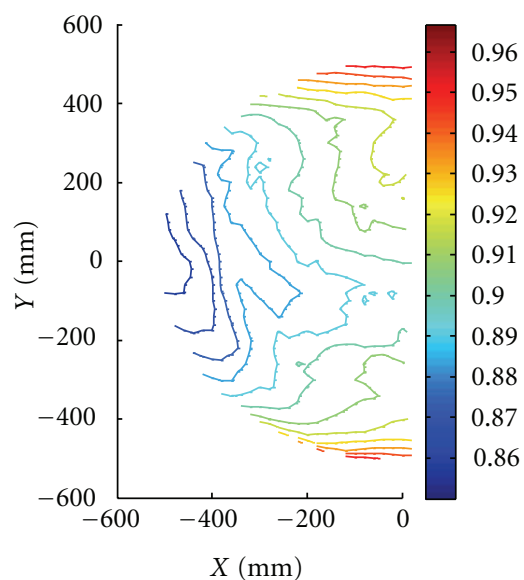

(b)

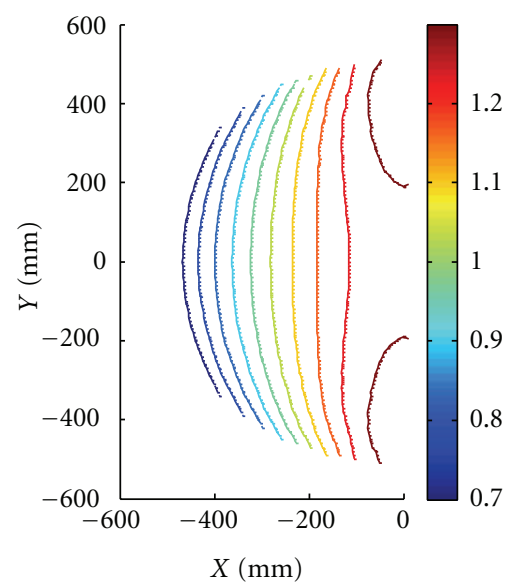

(c)

FIgURE 18: Manipulability index $\mu_{t}$ (a), normalized velocity along the $X$ direction (b), and DSI $\mu_{X}$ computed in the horizontal half-plane $X Y$ with $Z=-1050 \mathrm{~mm}$ (c). 


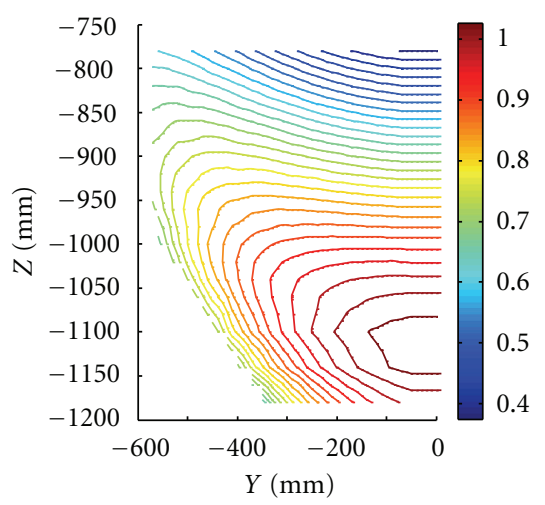

(a)

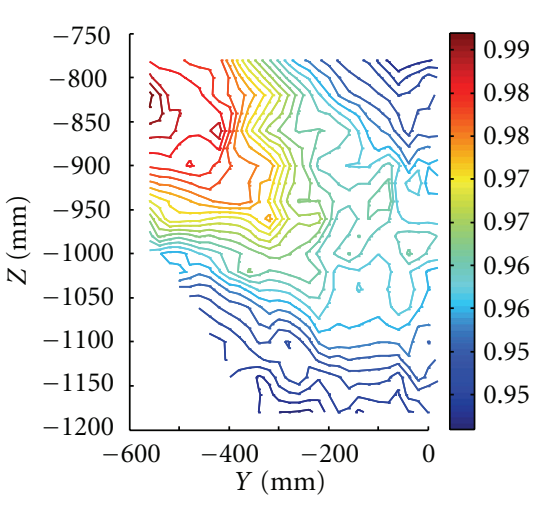

(b)

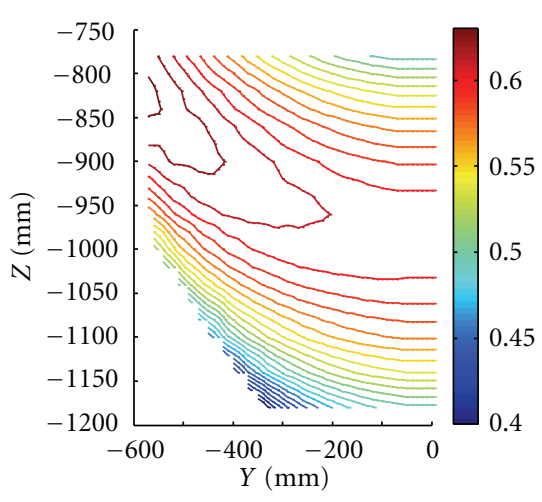

(c)

FIGURE 19: Manipulability index $\mu_{t}$ (a), normalized velocity along the $Z$ direction (b), and DSI $\mu_{Z}$ computed in the vertical half-plane $Y Z$ with $X=0 \mathrm{~mm}$ (c).

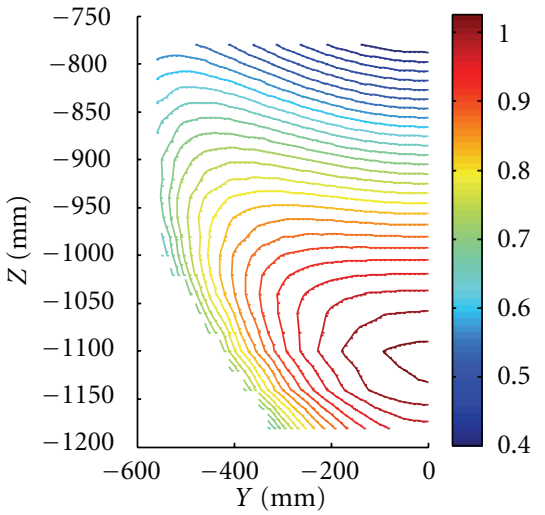

(a)

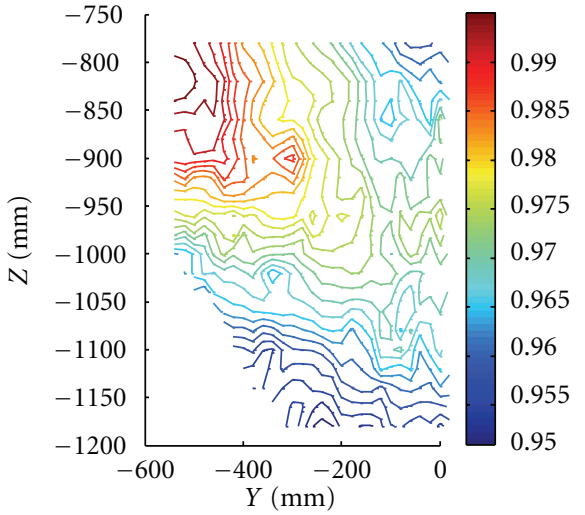

(b)

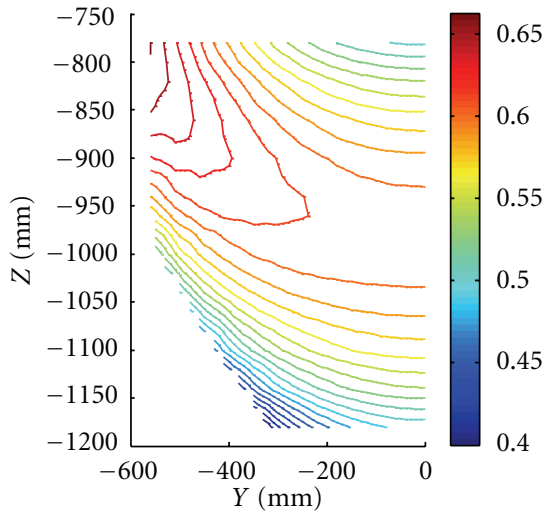

(c)

FIgURE 20: Manipulability index $\mu_{t}$ (a), normalized velocity along the $Z$ direction (b), and DSI $\mu_{Z}$ computed in the vertical half-plane $X Z$ with $X=100 \mathrm{~mm}$ (c).

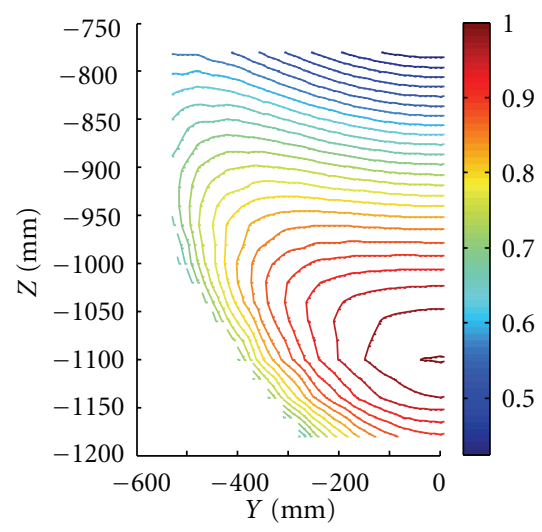

(a)

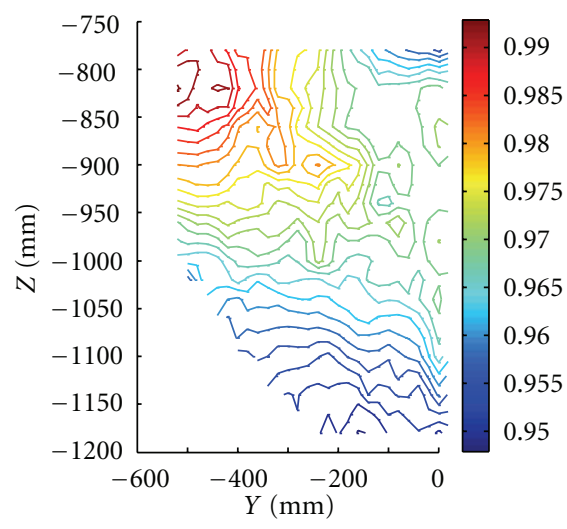

(b)

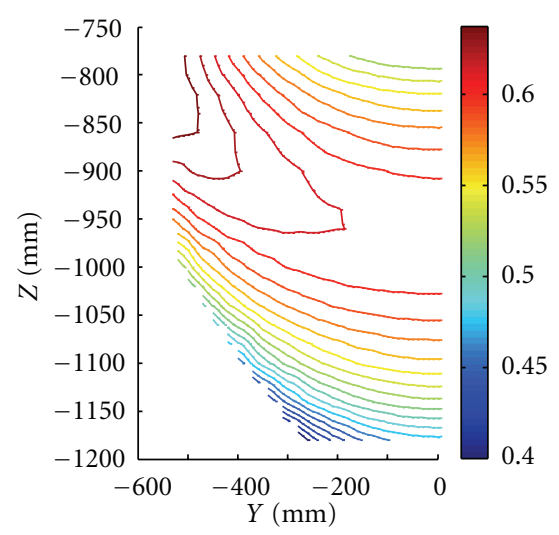

(c)

FIGURE 21: Manipulability index $\mu_{t}$ (a), normalized velocity along the $Z$ direction (b), and DSI $\mu_{Z}$ computed in the vertical half-plane $X Z$ with $X=200 \mathrm{~mm}$ (c). 


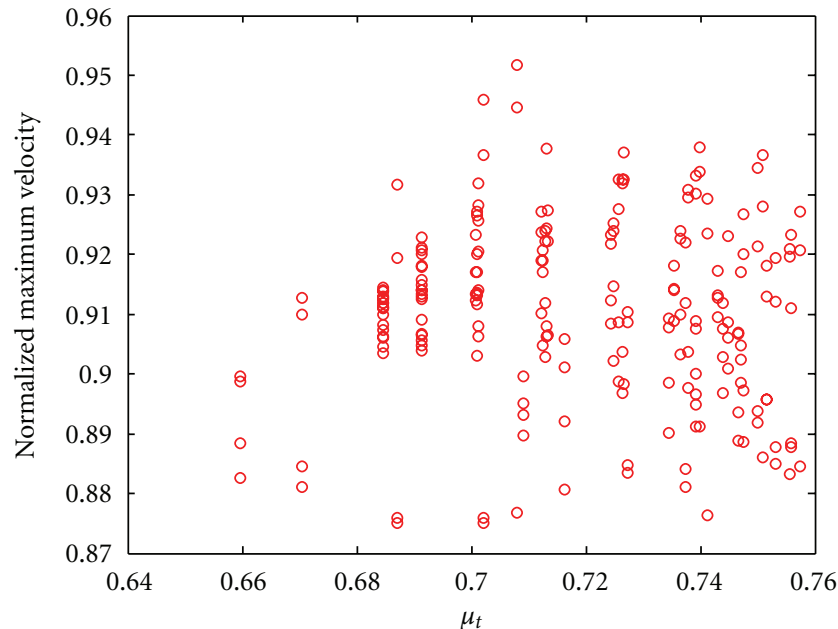

FIGURE 22: Scattered diagram of manipulability $\mu_{t}$ versus velocity along the $X$ direction (horizontal half-plane $X Y$ with $Z=$ $-900 \mathrm{~mm})$.

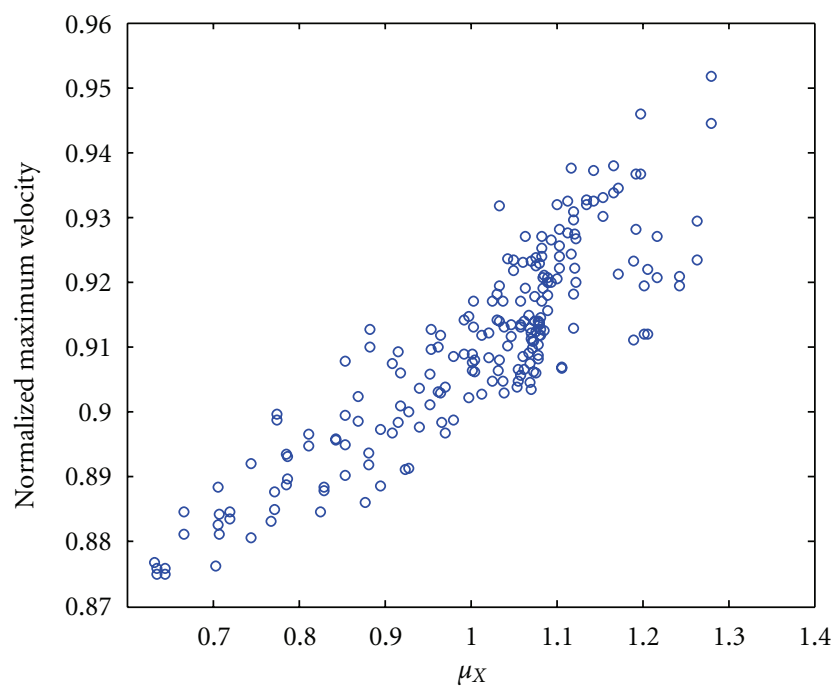

FIGURE 23: Scatter diagram of DSI $\mu_{X}$ versus velocity along the $X$ direction (horizontal half-plane $X Y$ with $Z=-900 \mathrm{~mm}$ ).

figures, while the DSIs $\mu_{X}$ and $\mu_{Z}$ are shown in the subplots (c).

It should be observed that in these figures the comparison among numerical and experimental data is restricted to halfplanes in consequence of the already proved symmetrical shapes of all the indexes.

It can be immediately recognized that in all cases the proposed DSIs $\mu_{X}$ and $\mu_{Z}$ show shapes very similar to the normalized maximum velocities. Not only do the best performance regions predicted by the DSIs fit well with the experimentally recorded data, but the performance variations foreseen by DSIs seem to be in good agreement with the recorded velocity variations. What is more important, the predictions provided by DSIs are considerably more accurate than the predictions provided by the manipulability index

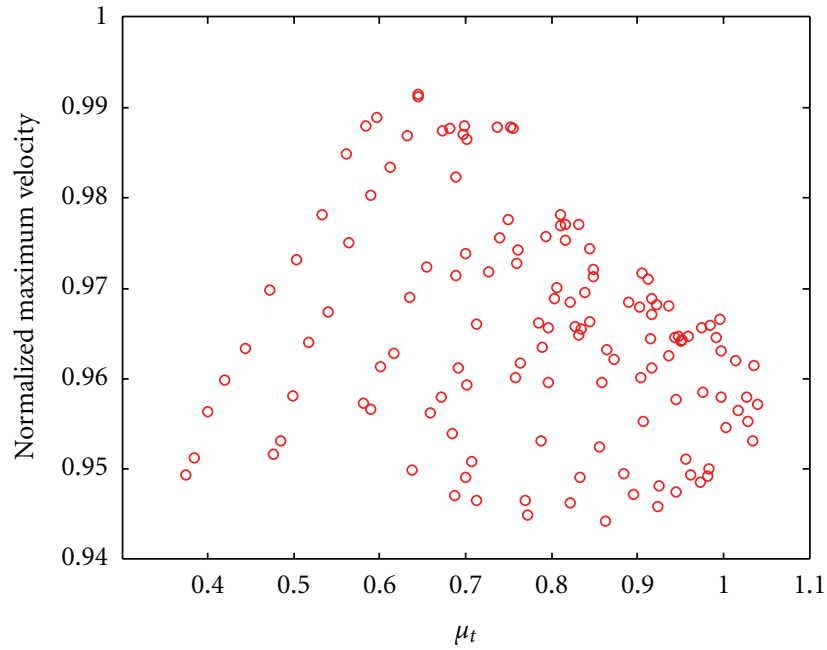

Figure 24: Scatter diagram of manipulability $\mu_{t}$ versus velocity along the $Z$ direction (vertical half-plane $Y Z$ with $X=0 \mathrm{~mm}$ ).

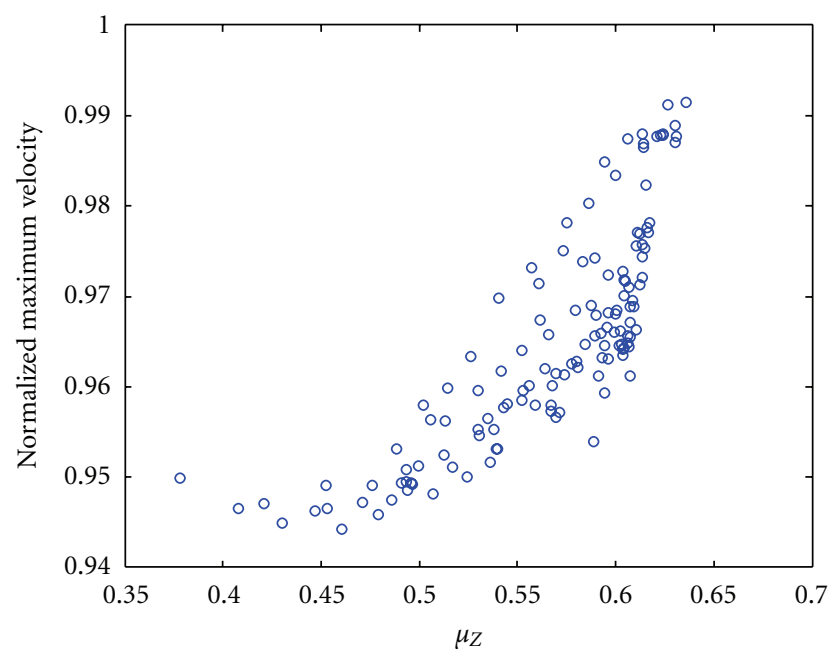

Figure 25: Scatter diagram of DSI $\mu_{Z}$ versus velocity along the $Z$ direction(vertical half-plane $Y Z$ with $X=0 \mathrm{~mm}$ ).

$\mu_{t}$. For example, in the case of horizontal displacements at $Z=-900 \mathrm{~mm}$ (Figure 17), $\mu_{t}$ suggests an inexistent best performance region at the center left of the halfplane (across the $X$ axis), while in the case of vertical displacements, regardless of the plane, it completely misses the best performance region locations.

As final evidence of the usefulness of the suggested DSIs, in the Figures from 22 through $25, \mu_{t}, \mu_{X}$, and $\mu_{Z}$ have been related to the normalized maximum velocities achieved at each grid point of the vertical and horizontal half-planes shown in Figures 17 and 19. Generally speaking, though the definitions of the adopted indexes are purely kinematic, it would be informative for good performance indexes to provide satisfactory estimates of dynamic performance variations within the workspace. In other words, a performance index would be practically useful if, for example, an increase of the robot velocity could be predicted through a 
corresponding increase of the performance index. Figures 22 and 24 show that this is not the case when manipulability is considered; there seems to be no meaningful correlation between $\mu_{t}$ and the end-effector velocities in the horizontal and vertical directions. On the contrary, Figures 23 and 25 prove that data are less scattered when the DSIs $\mu_{X}$ and $\mu_{Z}$ are considered; a satisfactory correlation appears between the DSIs and the velocity values.

\section{Conclusions}

Direction-selective indexes (DSIs) have been proposed to provide uncoupled evaluations of robot translational performances along specific directions. Such indexes, which might be considered an extension of the traditional manipulability index, overcome the limitations of manipulability (but also of other popular indexes such as the minimum singular value and the condition number of the Jacobian matrix) which just provide global evaluations of the robot capabilities that are often of limited practical usefulness.

The experimental investigation carried out on a suitably instrumented Adept Quattro commercial robot has proved that the proposed DSI formulation can provide reliable predictions of the robot performances in making movements along specific directions. In particular, the comparison among the values of DSIs $\left(\mu_{X}\right.$ and $\left.\mu_{Z}\right)$, manipulability $\mu_{t}$, and the normalized maximum velocities experimentally recorded performing horizontal and vertical movements in a large number of points has demonstrated that DSIs can better foresee the best performance regions within the workspace and that the performance variation predictions made through the DSIs are in good agreement with the recorded velocity variations. Overall the predictions made through the DSIs are considerably more accurate than the predictions provided by manipulability indexes.

So far, the effectiveness of DSIs has been assessed on a single family of parallel manipulators (4-RUU), but the results achieved are thought to have a general relevance; DSI definition just recourses to vectors extracted by the inverse Jacobian matrix and includes no constraints accounting for a specific parallel robot architecture. The proposed indexes can therefore be applied to any parallel robot architecture, as long as an inverse Jacobian matrix can be computed.

In conclusion, DSIs, though being purely kinematic indexes, may provide useful hints in foreseeing the robot dynamic performances along relevant directions of motion within the workspace. At robot design, installation, or programming stages, such information could be usefully employed to optimize the robot geometrical features, the robot location within a workcell, the location of the target frames with respect to the robot, and also the robot endeffector paths. All these considerations make the DSIs a useful tool for robot designers, manufacturers, and programmers.

\section{Acknowledgment}

The authors acknowledge the financial support of the FSU foundation ("Fondazione Studi Universitari"-Vicenza) through a Ph. D. scholarship and significant equipment grants.

\section{References}

[1] J. K. Salisbury and J. J. Craig, "Articulated hands: force control and kinematic issues," International Journal of Robotics Research, vol. 1, no. 1, pp. 4-17, 1982.

[2] T. Yoshikawa, "Manipulability of robotic mechanisms," International Journal of Robotics Research, vol. 4, no. 2, pp. 3-9, 1985.

[3] C. Gosselin and J. Angeles, "Global performance index for the kinematic optimization of robotic manipulators," Transaction of the ASME, Journal of Mechanical Design, vol. 113, no. 3, pp. 220-226, 1991.

[4] J. P. Merlet, "Jacobian, manipulability, condition number, and accuracy of parallel robots," Journal of Mechanical Design, Transactions of the ASME, vol. 128, no. 1, pp. 199-206, 2006.

[5] J. Angeles and C. S. Lopez-Cajun, "Kinematic isotropy and the conditioning index of serial robotic manipulators," International Journal of Robotics Research, vol. 11, no. 6, pp. 560-571, 1992.

[6] C. M. Gosselin, "The optimum design of robotic manipulators using dexterity indices," Robotics and Autonomous Systems, vol. 9, no. 4, pp. 213-226, 1992.

[7] S. G. Kim and J. Ryu, "New dimensionally homogeneous Jacobian matrix formulation by three end-effector points for optimal design of parallel manipulators," IEEE Transactions on Robotics and Automation, vol. 19, no. 4, pp. 731-737, 2003.

[8] S. G. Kim and J. Ryu, "Force transmission analyses with dimensionally homogeneous jacobian matrices for parallel manipulators," KSME International Journal, vol. 18, no. 5, pp. 780-788, 2004.

[9] G. Pond and J. A. Carretero, "Formulating Jacobian matrices for the dexterity analysis of parallel manipulators," Mechanism and Machine Theory, vol. 41, no. 12, pp. 1505-1519, 2006.

[10] R. V. Mayorga and J. Carrera, "A manipulator performance index based on the jacobian rate of change: a motion planning analysis," in Proceedings of the IEEE International Conference on Robotics and Automation, Orlando, Fla, USA, May 2006.

[11] Y. Li and Q. Xu, "A new approach to the architecture optimization of a general 3-PUU translational parallel manipulator," Journal of Intelligent and Robotic Systems: Theory and Applications, vol. 46, no. 1, pp. 59-72, 2006.

[12] Q. Xu and Y. Li, "An investigation on mobility and stiffness of a 3-DOF translational parallel manipulator via screw theory," Robotics and Computer-Integrated Manufacturing, vol. 24, no. 3, pp. 402-414, 2008.

[13] C. Gosselin, "Stiffness mapping for parallel manipulators," IEEE Transactions on Robotics and Automation, vol. 6, no. 3, pp. 377-382, 1990.

[14] Y. Li and Q. Xu, "Kinematic analysis and design of a new 3DOF translational parallel manipulator," Journal of Mechanical Design, Transactions of the ASME, vol. 128, no. 4, pp. 729-737, 2006.

[15] I. Mansouri and M. Ouali, "A new homogeneous manipulability measure of robot manipulators, based on power concept," Mechatronics, vol. 19, no. 6, pp. 927-944, 2009.

[16] I. Mansouri and M. Ouali, "The power manipulability-a new homogeneous performance index of robot manipulators," 
Robotics and Computer-Integrated Manufacturing, vol. 27, no. 2, pp. 434-449, 2011.

[17] J. Wang, X. Liu, and C. Wu, "Optimal design of a new spatial 3DOF parallel robot with respect to a frame-free index," Science in China E, vol. 52, no. 4, pp. 986-999, 2009.

[18] J. Wang, C. Wu, and X. J. Liu, "Performance evaluation of parallel manipulators: motion/force transmissibility and its index," Mechanism and Machine Theory, vol. 45, pp. 14621476, 2010.

[19] G. Boschetti and A. Trevisani, "Direction selective performance indexes for parallel manipulators," in Proceedings of the 1st Joint International Conference on Multibody System Dynamics, Lappeenranta, Finland, May 2010.

[20] O. Ma and J. Angeles, "Optimum architecture design of platform manipulator," in Proceedings of the IEEE International Conference on Robotics Automation, pp. 1131-1135, 1991.

[21] O. Company, F. Pierrot, S. Krut, and V. Nabat, "Simplified dynamic modeling and improvement of a four-degree-offreedom pick-and-place manipulator with articulated moving platform," Proceedings of IMechE Part I, vol. 223, no. 1, pp. 1327, 2009. 

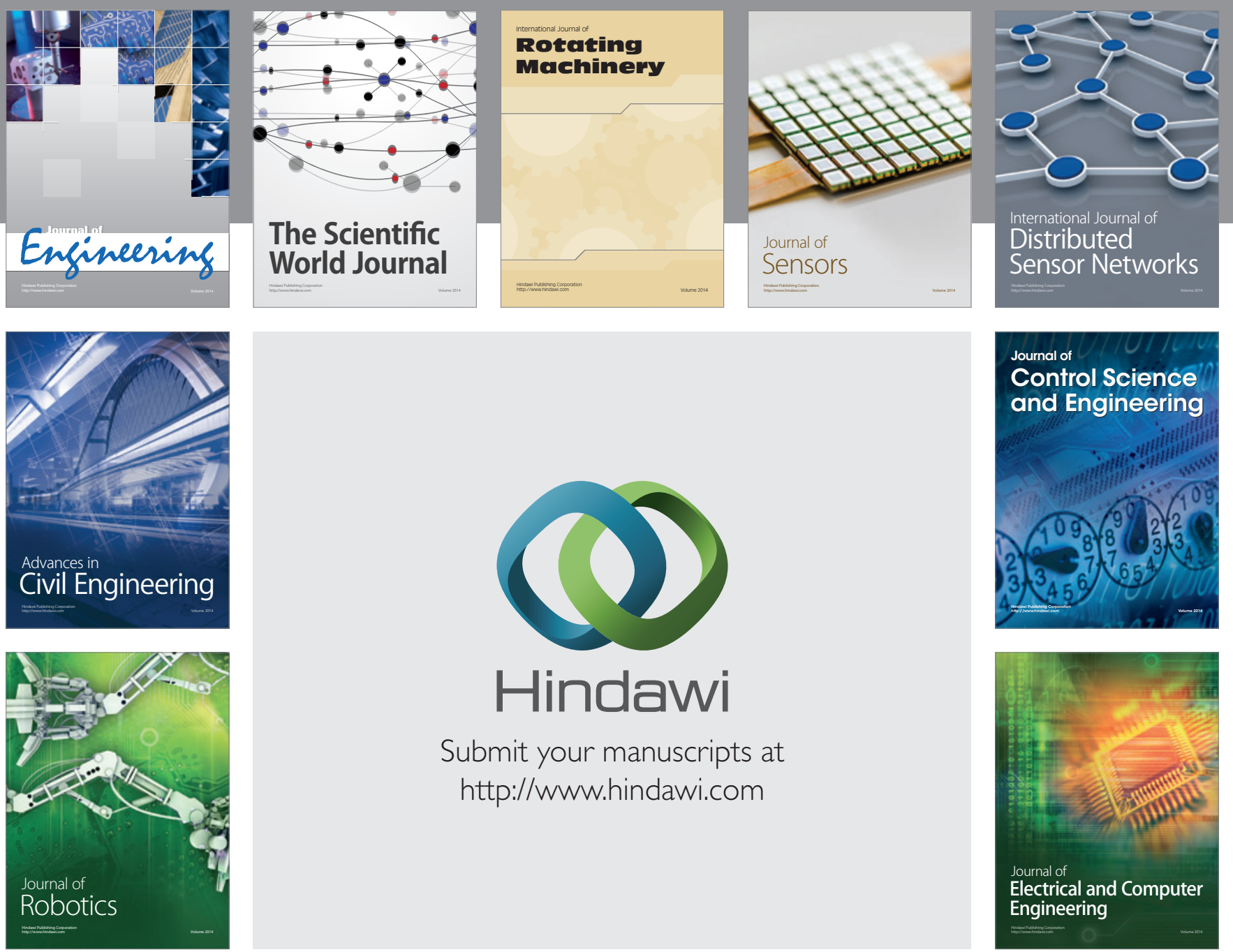

Submit your manuscripts at

http://www.hindawi.com
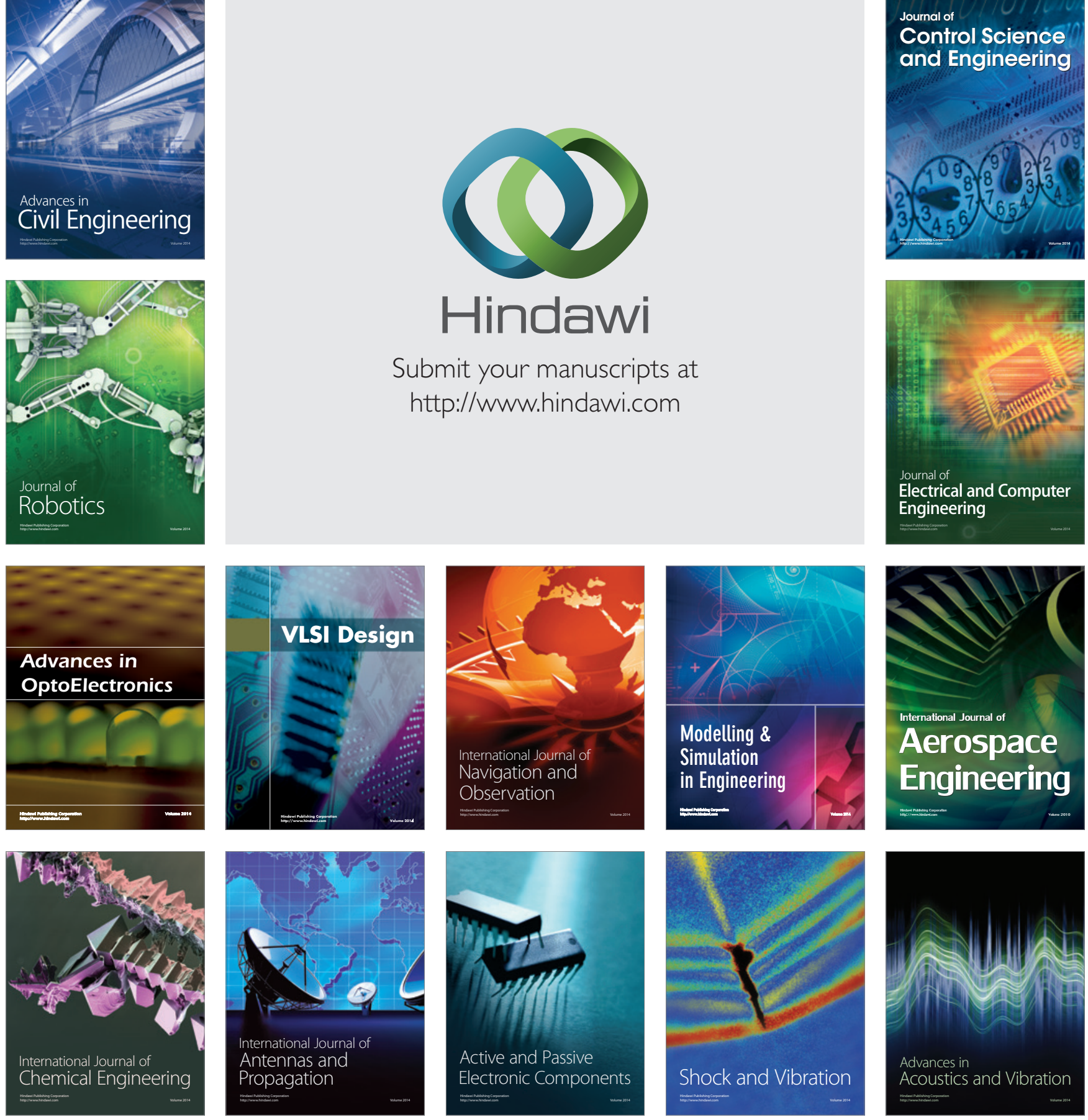